\title{
Decoding Heat Capacity Features from the Energy Landscape
}

\author{
David J. Wales \\ University Chemical Laboratories, University of Cambridge, \\ Lensfield Road, Cambridge CB2 1EW, U.K.
}

\begin{abstract}
A general scheme is derived to connect transitions in configuration space with features in the heat capacity. A formulation in terms of occupation probabilities for local minima that define the potential energy landscape provides a quantitative description of how contributions arise from competition between different states. The theory does not rely on a structural interpretation for the local minima, so it is equally applicable to molecular energy landscapes and the landscapes defined by abstract functions. Applications are presented for low temperature solid-solid transitions in atomic clusters, which involve just a few local minima with different morphologies, and for cluster melting, which is driven by the landscape entropy associated with the more numerous high energy minima. Analysing these features in terms of the balance between states with increasing and decreasing occupation probabilities provides a direct interpretation of the underlying transitions. This approach enables us to identify a qualitatively different transition that is caused by a single local minimum associated with an exceptionally large catchment volume in configuration space.
\end{abstract}

PACS numbers: 64.70.Nd, 36.40.Ei, 65.40.Ba, 64.60.-i, 05.70.Fh 
For atomic and molecular clusters, analysis of heat capacity features in terms of finite system analogues of phase transitions has proved particularly insightful. Clusters that exhibit a low temperature heat capacity peak corresponding to a solid-solid transition between alternative low energy morphologies are especially interesting [1-11]. The corresponding potential energy landscapes exhibit a double funnel structure, which leads to broken ergodicity. Solid-solid transitions generally involve just a few distinct low energy minima from the competing funnels [1-11]. It is usually possible to locate the relevant structures quite easily using global optimisation techniques, such as basin-hopping $[1,12,13]$. In contrast, melting transitions involve a relatively small number of low energy minima and a much larger ensemble of high energy minima favoured by entropy.

In the present contribution we provide a general theoretical framework that quantifies the contributions of local minima (or more general regions of configuration space) to features in the heat capacity. We express the heat capacity in terms of occupation probabilities and their temperature derivatives, and hence identify the states that define the underlying transition. This approach does not rely on assignments based on molecular structure, and we can therefore apply it to landscapes defined by abstract functions $[14,15]$. Applications to solid-solid transitions and melting in atomic clusters illustrate how the occupation probability analysis pinpoints the key states involved. The final example is a qualitatively different transition, where one particular local minimum becomes stabilised entropically at higher temperature. In a molecular system, this scenario could arise for an isomer with one or more very low frequency vibrational modes, corresponding to weakly coupled domains.

In the superposition approach $[6,11,16-19]$ we obtain an expression for the global partition function as a sum over contributions from local minima:

$$
Z(T)=\sum_{\gamma} Z_{\gamma}(T)
$$

This formulation reproduces the equilibrium occupation probability for each minimum as a function of temperature, and in this sense it is explicitly ergodic. Normal mode analysis for each minimum yields a harmonic approximation to the vibrational density of states, which is quite accurate at low temperature $[10,11]$. The partition function for minimum $\alpha$ is

$$
Z_{\alpha}(T)=\frac{2 \prod_{s} N_{s} !}{o_{\alpha}}\left(\frac{k_{B} T}{h \bar{\nu}_{\alpha}}\right)^{\kappa} e^{-V_{\alpha} / k_{B} T} \equiv n_{\alpha}\left(\frac{k_{B} T}{h \bar{\nu}_{\alpha}}\right)^{\kappa} e^{-V_{\alpha} / k_{B} T}
$$

where $N_{s}$ is the number of atoms of element $s$ with $\sum_{s} N_{s}=N, o_{\alpha}$ is the order of the molecular point group, $k_{B}$ is the Boltzmann constant, $h$ is the Planck constant, $\bar{\nu}_{\alpha}$ and $V_{\alpha}$ are the geometric 
mean normal mode vibrational frequency and potential energy of minimum $\alpha$, and $\kappa=3 N-6$ is the number of vibrational degrees of freedom. Corrections for well anharmonicity can be included $[17,20-23]$. In the present contribution accurate anharmonic densities of states are employed from basin-sampling calculations [24].

The internal energy and heat capacity corresponding to the superposition partition function are:

$$
\begin{gathered}
E=k_{B} T^{2}\left(\frac{\partial \ln Z(T)}{\partial T}\right)_{N, V}=\kappa k_{B} T+\frac{1}{Z(T)} \sum_{\gamma} n_{\gamma} V_{\gamma}\left(\frac{k_{B} T}{h \bar{\nu}_{\gamma}}\right)^{\kappa} e^{-V_{\gamma} / k_{B} T} \equiv \kappa k_{B} T+\frac{z_{1}(T)}{z_{0}(T)}, \\
\text { and } C_{V}=\left(\frac{\partial E}{\partial T}\right)_{N, V}=\kappa k_{B}-\frac{z_{1}(T)^{2}}{k_{B} T^{2} z_{0}(T)^{2}}+\frac{z_{2}(T)}{k_{B} T^{2} z_{0}(T)}, \\
\text { with } \quad z_{r}(T)=\sum_{\gamma} n_{\gamma}\left(V_{\gamma}\right)^{r}\left(\frac{k_{B} T}{h \bar{\nu}_{\gamma}}\right)^{\kappa} e^{-V_{\gamma} / k_{B} T} .
\end{gathered}
$$

To obtain the configurational contribution we can subtract $\kappa k_{B} T / 2$ from $E$ and $\kappa k_{B} / 2$ from $C_{V}$.

For the harmonic superposition partition function the occupation probability for minimum $\alpha$ is

$$
p_{\alpha}(T)=\frac{n_{\alpha} e^{-V_{\alpha} / k_{B} T} / \bar{\nu}_{\alpha}^{\kappa}}{\sum_{\gamma} n_{\gamma} e^{-V_{\gamma} / k_{B} T} / \bar{\nu}_{\gamma}^{\kappa}} .
$$

From the definitions we have

$$
\begin{gathered}
z_{r}(T)=Z(T) \sum_{\gamma} p_{\gamma}(T)\left(V_{\gamma}\right)^{r}=z_{0}(T) \sum_{\gamma} p_{\gamma}(T)\left(V_{\gamma}\right)^{r}, \\
\text { and } \quad \begin{aligned}
\frac{\partial p_{\alpha}(T)}{\partial T} & =-\frac{p_{\alpha}(T)}{Z(T)} \frac{\partial Z(T)}{\partial T}+\frac{\kappa p_{\alpha}(T)}{T}+\frac{p_{\alpha}(T) V_{\alpha}}{k_{B} T^{2}} \\
& =-p_{\alpha}(T) \frac{\partial \ln Z(T)}{\partial T}+\frac{\kappa p_{\alpha}(T)}{T}+\frac{p_{\alpha}(T) V_{\alpha}}{k_{B} T^{2}} .
\end{aligned}
\end{gathered}
$$

Since $k_{B} T^{2} \partial \ln Z(T) / \partial T=E=\kappa k_{B} T+\sum_{\gamma} p_{\gamma}(T) V_{\gamma}$ we obtain

$$
\begin{aligned}
g_{\alpha}(T) \equiv \frac{\partial p_{\alpha}(T)}{\partial T} & =p_{\alpha}\left(\frac{\kappa}{T}+\frac{V_{\alpha}}{k_{B} T^{2}}-\frac{\kappa}{T}-\frac{1}{k_{B} T^{2}} \sum_{\gamma} p_{\gamma}(T) V_{\gamma}\right) \\
& =\frac{p_{\alpha}}{k_{B} T^{2}}\left(V_{\alpha}-\sum_{\gamma} p_{\gamma}(T) V_{\gamma}\right) \equiv \frac{p_{\alpha}}{k_{B} T^{2}}\left(V_{\alpha}-\langle V\rangle_{\min }\right) .
\end{aligned}
$$

Hence minima with energies above and below the mean value $\langle V\rangle_{\min }$ have increasing and decreasing occupation probabilities, respectively, as $T$ increases. From equations (3) and (6)

$$
C_{V}=\kappa k_{B}-\frac{\left(\sum_{\gamma} p_{\gamma}(T) V_{\gamma}\right)^{2}}{k_{B} T^{2}\left(\sum_{\gamma} p_{\gamma}(T)\right)^{2}}+\frac{\sum_{\gamma} p_{\gamma}(T)\left(V_{\gamma}\right)^{2}}{k_{B} T^{2} \sum_{\gamma} p_{\gamma}(T)}=\kappa k_{B}+\frac{\left\langle V^{2}\right\rangle_{\min }-\langle V\rangle_{\min }^{2}}{k_{B} T^{2}} .
$$


Equation (8) can be used to obtain an alternative expression for the potential energy fluctuation:

$$
\begin{aligned}
\sum_{\gamma} g_{\gamma}(T)\left(V_{\gamma}-\langle V\rangle_{\min }\right) & =\sum_{\gamma} \frac{p_{\gamma}}{k_{B} T^{2}}\left(V_{\gamma}-\langle V\rangle_{\min }\right)^{2} \\
=\frac{1}{k_{B} T^{2}} \sum_{\gamma} p_{\gamma}\left[\left(V_{\gamma}\right)^{2}+\langle V\rangle_{\min }^{2}-2\langle V\rangle_{\min } V_{\gamma}\right] & =\frac{\left\langle V^{2}\right\rangle_{\min }-\langle V\rangle_{\min }^{2}}{k_{B} T^{2}} .
\end{aligned}
$$

Hence we can relate the heat capacity to occupation probability gradients as

$$
\begin{aligned}
C_{V} & =\kappa k_{B}+\sum_{\gamma} g_{\gamma}(T)\left(V_{\gamma}-\langle V\rangle_{\min }\right) \\
& =\kappa k_{B}+k_{B} T^{2} \sum_{\gamma} g_{\gamma}(T)^{2} \frac{1}{p_{\gamma}(T)}=\kappa k_{B}+k_{B} T^{2} \sum_{\gamma} g_{\gamma}(T)\left(\frac{\partial \ln p_{\gamma}(T)}{\partial T}\right) .
\end{aligned}
$$

A local maximum in $C_{V}(T)$ is therefore equivalent to a maximum in $\sum_{\gamma} g_{\gamma}(T)\left(\partial \ln p_{\gamma}(T) / \partial T\right)$. Since $\partial \ln p_{\gamma}(T) / \partial T$ has the same sign as $g_{\gamma}(T)$ we can interpret such peaks in terms of the contributions from local minima with positive and negative temperature derivatives:

$$
\begin{aligned}
C_{V} & =\kappa k_{B}+\sum_{\gamma}^{g_{\gamma}(T)>0} g_{\gamma}(T)\left(V_{\gamma}-\langle V\rangle_{\min }\right)+\sum_{\gamma}^{g_{\gamma}(T)<0} g_{\gamma}(T)\left(V_{\gamma}-\langle V\rangle_{\min }\right) \\
& \equiv \kappa k_{B}+C^{+}(T)+C^{-}(T),
\end{aligned}
$$

For a decomposition within the normal mode approximation this analysis is sufficient to determine which local minima are primarily responsible for any given heat capacity feature. Hence we have an unbiased assignment of contributions to $C_{V}(T)$. For example, the landscape defined by the cost function involved in fitting a neural network depends upon weights between nodes [15].

To exploit the present analysis for visualisation we set a threshold for the contributions to $C^{ \pm}$ at the temperature of interest, $T^{*}$, adding them in order of decreasing magnitude. The minima that contribute a given fraction of the total $C^{ \pm}\left(T^{*}\right)$ are then coloured in the corresponding disconnectivity graph $[25,26]$. Details of how these graphs are constructed can be found elsewhere $[6,25,26]$. In brief, databases of local minima and the transition states that connect them are obtained using well established methods based on geometry optimisation [6]. To visualise the resulting kinetic transition networks, local minima are associated with the termini of branches on a vertical energy scale. At a regular series of energy thresholds, branches are joined if the corresponding minima can intervert via a pathway where the highest transition state lies below the threshold. The organisation on the horizontal axis was chosen to prevent branches from crossing, which produces a hierarchical grouping of sets that merge at increasing energy thresholds. 
In each example presented below, minima with positive and negative $g_{\alpha}\left(T^{*}\right)$ contributing up to a fraction $f$ for $C^{+}\left(T^{*}\right)$ and $C^{-}\left(T^{*}\right)$ have branches coloured blue and red. If $f=1$ then all branches will be either red or blue. However, for low temperature peaks in $C_{V}$ only a few local minima have significant occupation probabilities. Choosing a cutoff at $f=0.9$ or 0.99 highlights these states. It is then meaningful to associate the heat capacity feature with a transition between these sets. This assignment could also be used to define products and reactants in the calculation of overall rate constants.

The heat capacity features that appear at higher temperature in atomic clusters are associated with the finite system analogue of melting. For small systems, such as $\mathrm{LJ}_{13}$ and $\mathrm{LJ}_{14}$, it is straightforward to obtain practically complete sets of local minima. If $C_{V}(T)$ is then calculated using harmonic vibrational densities of states the melting peak is systematically shifted to higher temperature (Figure 1), because the higher energy minima in the high entropy liquid-like phase generally have more anharmonic vibrational modes. Hence the harmonic analysis for local minima can account for the landscape entropy (potential energy density of local minima), but is missing the anharmonic contributions to these well entropies.

For large systems it is not possible to characterise sufficient local minima in the liquid-like phase to account for the landscape entropy directly. Instead, we must adopt an appropriate sampling scheme. Here we employ the basin-sampling approach, which can treat the broken ergodicity associated with multifunnel landscapes very efficiently, producing quantitatively accurate densities of states [24]. This method couples basin-hopping global optimisation to locate low-lying minima, with parallel tempering at high temperature. A two-dimensional distribution is calculated for the instantaneous potential energy $V^{I}$ and the quench energy obtained on local minimisation, $V^{Q}$. A common energy bin width $\Delta$ was chosen, and the bins are indexed by subscripts $i$ and $q$ as $V_{i}^{I}$ and $V_{q}^{Q}$.

Optimal one- and two-dimensional weights $W_{i}^{1 D}$ and $W_{i q}^{2 D}$ are obtained from overlapping distributions corresponding to replicas at different temperatures by direct minimisation of $\chi^{2}$ statistics [24]. For $W_{i q}^{2 D}$ an anharmonic fit is used for each $Q$ bin [24]. The occupation probability of instantaneous potential energy bin $i$ at temperature $T, p_{i}(T)$ is -147

$$
p_{i}(T)=\frac{W_{i}^{1 D} e^{-V_{i}^{I} / k_{B} T}}{\sum_{j}^{\mathrm{I} \text { bins }} W_{j}^{1 D} e^{-V_{j}^{I} / k_{B} T}}=\frac{e^{-V_{i}^{I} / k_{B} T} \sum_{q}^{\mathrm{Q} \text { bins }} W_{i q}^{2 D}}{\sum_{j}^{\mathrm{I} \text { bins }} e^{-V_{j}^{I} / k_{B} T} \sum_{r}^{\mathrm{Q} \text { bins }} W_{j r}^{2 D}} .
$$


For bin width $\Delta$ the microcanonical configurational density of states $\Omega_{c}\left(V^{I}\right)$ is proportional to

$$
\sum_{j}^{\text {I bins }} W_{j}^{1 D} \Theta\left(V^{I}-V_{j}^{I}\right) \Theta\left(V_{j}^{I}+\Delta-V^{I}\right) .
$$

Laplace transform gives the configurational part of $Z(T)$

$$
Z_{c}(T)=k_{B} T\left(1-e^{-\Delta / k_{B} T}\right) \sum_{j}^{\mathrm{I} \text { bins }} W_{j}^{1 D} e^{-V_{j}^{I} / k_{B} T} .
$$

The temperature derivatives of the occupation probabilities are

$$
g_{i}(T) \equiv \frac{\partial p_{i}(T)}{\partial T}=\frac{p_{i}}{k_{B} T^{2}}\left(V_{i}^{I}-\left\langle V^{I}\right\rangle_{\mathrm{I} \text { bins }}\right),
$$

where $\left\langle V^{I}\right\rangle_{\text {I bins }}=\sum_{j}^{\mathrm{I} \text { bins }} p_{j}(T) V_{j}^{I}$. Adding $\kappa k_{B} / 2$ to the configurational heat capacity we then obtain various equivalent expressions for $C_{V}(T)$

$$
C_{V}=\frac{\kappa k_{B}}{2}+k_{B}-\frac{\Delta^{2} e^{\Delta / k_{B} T}}{k_{B} T^{2}\left(1-e^{\Delta / k_{B} T}\right)^{2}}+\left\{\begin{array}{l}
k_{B} T^{2} \sum_{j}^{\mathrm{I} \text { bins }} g_{i}(T)^{2} \frac{1}{p_{j}(T)}, \\
k_{B} T^{2} \sum_{j}^{\mathrm{I} \text { bins }} g_{i}(T)\left(\frac{\partial \ln p_{j}(T)}{\partial T}\right), \\
\sum_{j}^{\mathrm{I} \text { bins }} g_{i}(T)\left(V_{j}^{I}-\left\langle V^{I}\right\rangle_{\mathrm{I} \text { bins }}\right) .
\end{array}\right.
$$

Equation (17) can be used to analyse heat capacity features in terms of positive and negative temperature derivatives of potential energy bin occupation probabilities, analogous to the interpretations based on local minima above. It is also possible to project the above formulation onto contributions of potential energy minima by reordering the sums over $i$ and $q$ :

$$
\begin{aligned}
& \sum_{j}^{\mathrm{I} \text { bins }} g_{j}(T)\left(V_{j}^{I}-\left\langle V^{I}\right\rangle_{\mathrm{I} \text { bins }}\right)=\sum_{j}^{\mathrm{I} \text { bins }} \frac{p_{j}}{k_{B} T^{2}}\left(V_{j}^{I}-\left\langle V^{I}\right\rangle_{\mathrm{I} \text { bins }}\right)^{2} \\
& =\sum_{q}^{\mathrm{Q} \text { bins }}\left[\sum_{g_{j}(T)>0}^{\mathrm{I} \text { bins }} \frac{\left(V_{j}^{I}-\left\langle V^{I}\right\rangle_{\mathrm{I} \text { bins }}\right)^{2} W_{j q}^{2 D} e^{-V_{j}^{I} / k_{B} T}}{k_{B} T^{2} \sum_{m}^{\mathrm{I} \text { bins }} W_{m}^{1 D} e^{-V_{m}^{I} / k_{B} T}}+\sum_{g_{j}(T)<0}^{\mathrm{I} \text { bins }} \frac{\left(V_{j}^{I}-\left\langle V^{I}\right\rangle_{\mathrm{I} \text { bins }}\right)^{2} W_{j q}^{2 D} e^{-V_{j}^{I} / k_{B} T}}{k_{B} T^{2} \sum_{m}^{\mathrm{I} \text { bins }} W_{m}^{1 D} e^{-V_{m}^{I} / k_{B} T}}\right] \\
& \equiv \sum_{q}^{\mathrm{Q} \text { bins }}\left[c_{q}^{+}+c_{q}^{-}\right] .
\end{aligned}
$$


The contribution to $C_{V}(T)$ from quench bin $q$ can contain terms corresponding to $I$ bins with both positive and negative $g_{j}(T)$. To associate a quench bin with overall positive or negative probability gradient we therefore consider the overall contribution $c_{q}^{\text {diff }}\left(T^{*}\right)=c_{q}^{+}\left(T^{*}\right)-c_{q}^{-}\left(T^{*}\right)$. The sets of positive and negative $c_{q}^{\text {diff }}\left(T^{*}\right)$ are sorted according to $\left|c_{q}^{\text {diff }}\left(T^{*}\right)\right|$ and fractional contributions to $C_{V}(T)$ from the two signs are defined in terms of the sums over the two subsets. For visualisation, the local minima that lie in any given quench bin are associated with the fractional contribution to $c_{q}^{+}\left(T^{*}\right)$ or $c_{q}^{-}\left(T^{*}\right)$ (whichever is larger) for that bin.

The following applications illustrate the interpretation of heat capacity features for solid-solid and melting transitions in atomic clusters, along with an example for a neural network. Heat capacity plots are illustrated in Figure 1 for three clusters bound by the Lennard-Jones potential and for a machine learning landscape. The systematic shift of the melting peak to higher temperature in the harmonic approximation is shown for the $\mathrm{LJ}_{13}$ cluster in Figure 1a. The other cluster sizes, $\mathrm{LJ}_{31}$ and $\mathrm{LJ}_{75}$, exhibit low temperature solid-solid peaks [1-11], which are associated with slow relaxation time scales and broken ergodicity.

Figure 2 shows disconnectivity graphs for $\mathrm{LJ}_{13}$ and $\mathrm{LJ}_{14}$ comparing thresholds of $90 \%$ and 99\% for the heat capacity contributions, using breakdowns based on the harmonic superposition framework for $\mathrm{LJ}_{14}$ and basin-sampling for $\mathrm{LJ}_{13}$. In each case the transition is described in terms of a negative occupation probability gradient from the global minimum, with the positive gradient delocalised over numerous higher energy minima. This description is consistent with our usual interpretation in terms of competition between low energy, low entropy and high energy, high entropy phase-like forms. Interrogating how different local minima contribute to a given heat capacity peak provides detailed insight into the origin of these features [27].

For $\mathrm{LJ}_{31}$ and $\mathrm{LJ}_{75}$ disconnectivity graphs are presented in Figure 3 for states that contribute $99 \%$ of the peaks corresponding to the solid-solid and melting transitions. In each case, only the global minimum contributes significantly to the negative gradient component at the solidsolid transition, while 3 and 19 minima are identified with the positive gradient components, for $\mathrm{LJ}_{31}$ and $\mathrm{LJ}_{75}$, respectively. In contrast, for the two melting transitions the positive and negative gradient contributions to the heat capacity are delocalised over a significant fraction of all the minima in the corresponding databases. These results were obtained using densities of states from basin-sampling [24]. The two-dimensional distribution obtained in the basin-sampling approach produces an accurate anharmonic density of states by appropriate reweighting [24].

These results for molecular energy landscapes all provide quantitative support for interpreta- 
tions of the cluster thermodynamics based upon analysis of the families of structures that represent the competing phase-like forms. No such intuition is available for the machine learning landscape. The disconnectivity graph in Figure 4 is coloured according to the heat capacity contributions at the two temperatures corresponding to the maxima in the $C_{V}$ analogue function plotted in Figure 1b. In contrast to the molecular examples, both peaks are accounted for by a relatively small number of local minima, and we do not see any analogue of melting. Instead, the high temperature peak corresponds to occupation of a single minimum with exceptionally high entropy, associated with a low curvature degree of freedom. This scenario is qualitatively different from the cluster melting transitions, which are mostly driven by the much larger number of high energy minima (landscape entropy), with a smaller contribution from the local vibrational well entropy.

The present contribution shows how a formulation in terms of occupation probabilities for local minima, or states defined by potential energy bins, provides a quantitative assignment for heat capacity features that is applicable to landscapes beyond molecular science. For the atomic clusters, this approach reinforces our structural interpretation of how such features are encoded in the underlying potential energy surface. It also provides a way to visualise the competition between phase-like forms using disconnectivity graphs. These tools should lead to new insight into the behaviour of molecular systems, and allow us to make connections with more general landscapes, such as those defined by neural networks. Here there is the possibility of combining different fits to obtain more accurate predictions and classifications. It seems likely that combinations corresponding to qualitatively different local minima would be useful, and these are precisely the solutions highlighted by transitions in configuration space associated with features in the analogue of the heat capacity. Decomposing contributions to the heat capacity using occupation probabilities provides a direct way to obtain the corresponding minima.

Acknowledgements. I am very grateful to Dmitri Schebarchov and Florent Calvo for their comments on the manuscript. This research was supported by the EPSRC.

[1] D. J. Wales and J. P. K. Doye, J. Phys. Chem. A 101, 5111 (1997).

[2] J. P. K. Doye, D. J. Wales and M. A. Miller, J. Chem. Phys. 109, 8143 (1998).

[3] J. P. K. Doye, M. A. Miller and D. J. Wales, J. Chem. Phys. 110, 6896 (1999).

[4] F. Calvo, J. P. Neirotti, D. L. Freeman and J. D. Doll, J. Chem. Phys. 112, 10350 (2000). 
[5] J. P. Neirotti, F. Calvo, D. L. Freeman and J. D. Doll, J. Chem. Phys. 112, 10340 (2000).

[6] D. J. Wales, Energy Landscapes, Cambridge University Press, Cambridge (2003).

[7] P. A. Frantsuzov and V. A. Mandelshtam, Phys. Rev. E 72, 037102 (2005).

[8] C. Predescu, P. A. Frantsuzov and V. A. Mandelshtam, J. Chem. Phys. 122, 154305 (2005).

[9] H. B. Liu and K. D. Jordan, J. Phys. Chem. B 109, 5203 (2005).

[10] V. A. Sharapov and V. A. Mandelshtam, J. Phys. Chem. A 111, 10284 (2007).

[11] V. A. Sharapov, D. Meluzzi and V. A. Mandelshtam, Phys. Rev. Lett. 98, 105701 (2007).

[12] Z. Li and H. A. Scheraga, Proc. Natl. Acad. Sci. USA 84, 6611 (1987).

[13] D. J. Wales and H. A. Scheraga, Science 285, 1368 (1999).

[14] M. Pavlovskaia, K. Tu and S.-C. Zhu, arXiv:1410.0576 [stat.ML] (2014).

[15] A. J. Ballard, J. D. Stevenson, R. Das and D. J. Wales, J. Chem. Phys. 144, 124119 (2016).

[16] F. H. Stillinger and T. A. Weber, Science 225, 983 (1984).

[17] D. J. Wales, Mol. Phys. 78, 151 (1993).

[18] F. H. Stillinger, Science 267, 1935 (1995).

[19] B. Strodel and D. J. Wales, Chem. Phys. Lett. 466, 105 (2008).

[20] J. P. K. Doye and D. J. Wales, J. Chem. Phys. 102, 9659 (1995).

[21] J. P. K. Doye and D. J. Wales, J. Chem. Phys. 102, 9673 (1995).

[22] F. Calvo, J. P. K. Doye and D. J. Wales, J. Chem. Phys. 115, 9627 (2001).

[23] I. Georgescu and V. A. Mandelshtam, J. Chem. Phys. 137, 144106 (2012).

[24] D. J. Wales, Chem. Phys. Lett. 584, 1 (2013).

[25] O. M. Becker and M. Karplus, J. Chem. Phys. 106, 1495 (1997).

[26] D. J. Wales, M. A. Miller and T. R. Walsh, Nature 394, 758 (1998).

[27] B. E. Husic, D. Schebarchov and D. J. Wales, Nanoscale 8, 18326 (2016).

[28] J. P. K. Doye, M. A. Miller and D. J. Wales, J. Chem. Phys. 111, 8417 (1999). 


\section{Figures}
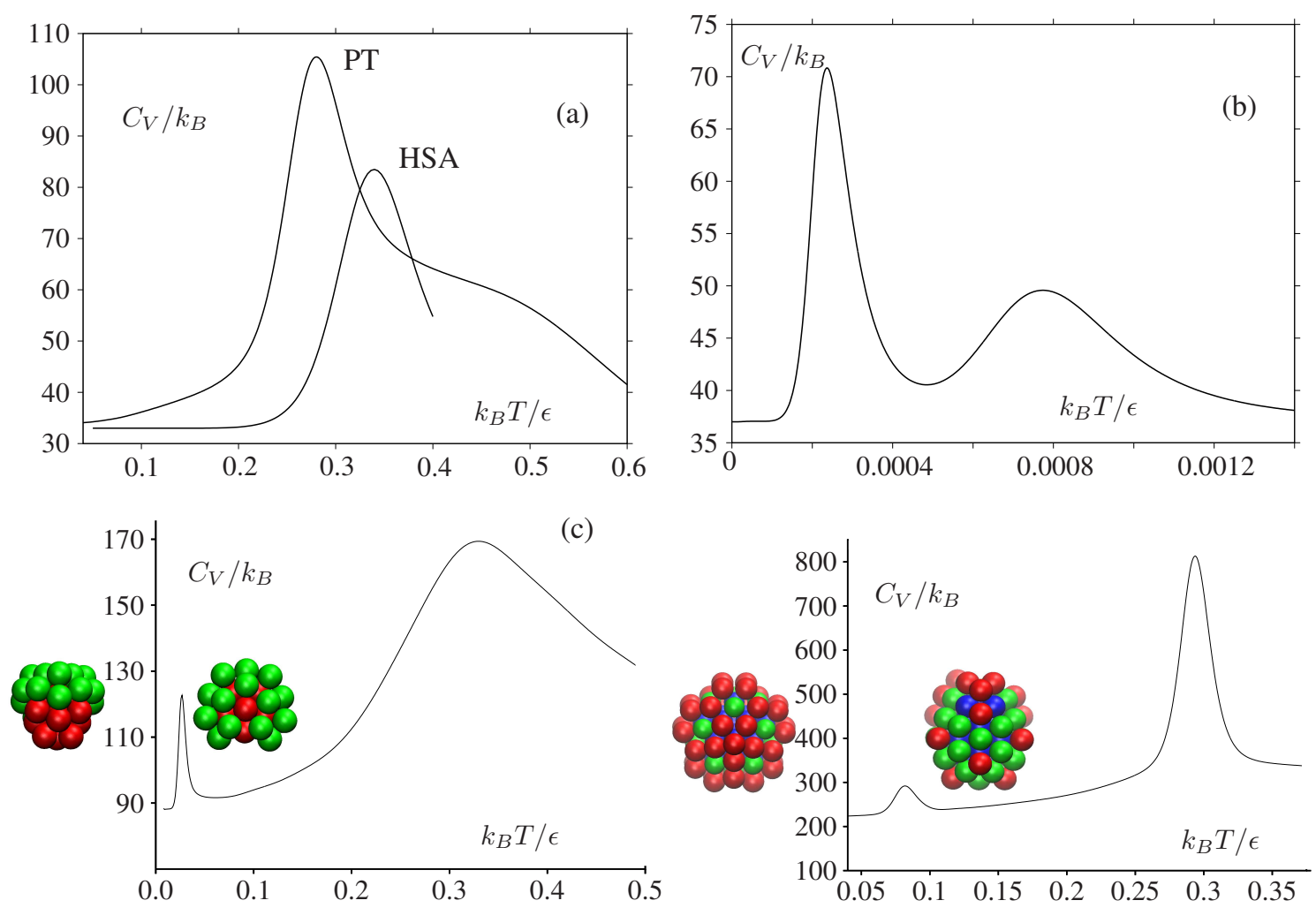

FIG. 1: (a) $C_{V}$ for $\mathrm{LJ}_{13}$ calculated using parallel tempering (PT) and the harmonic superposition approximation (HSA) $[6,11,16,17]$. (b) $C_{V}$ for the machine learning landscape obtained by fitting a three-layer neural network to predict patient outcomes from a combination of vital signs. (c) $C_{V}$ for $\mathrm{LJ}_{31}$ calculated using basin-sampling [24]. The lowest and second-lowest minima with Mackay and anti-Mackay overlayers [28] coloured in green are illustrated on the left and right of the low-temperature peak, respectively. The 13atom icosahedral core is coloured red in both structures. (d) $C_{V}$ for $\mathrm{LJ}_{75}$ calculated using basin-sampling [24]. The lowest and second-lowest minima based on a Marks decahedron and an incomplete Mackay icosahedron are illustrated on the left and right of the low-temperature peak, respectively. The atoms are coloured according to their contribution to the total energy: the most tightly bound atoms are blue, the least tightly bound are red, with intermediate binding energies in green. 

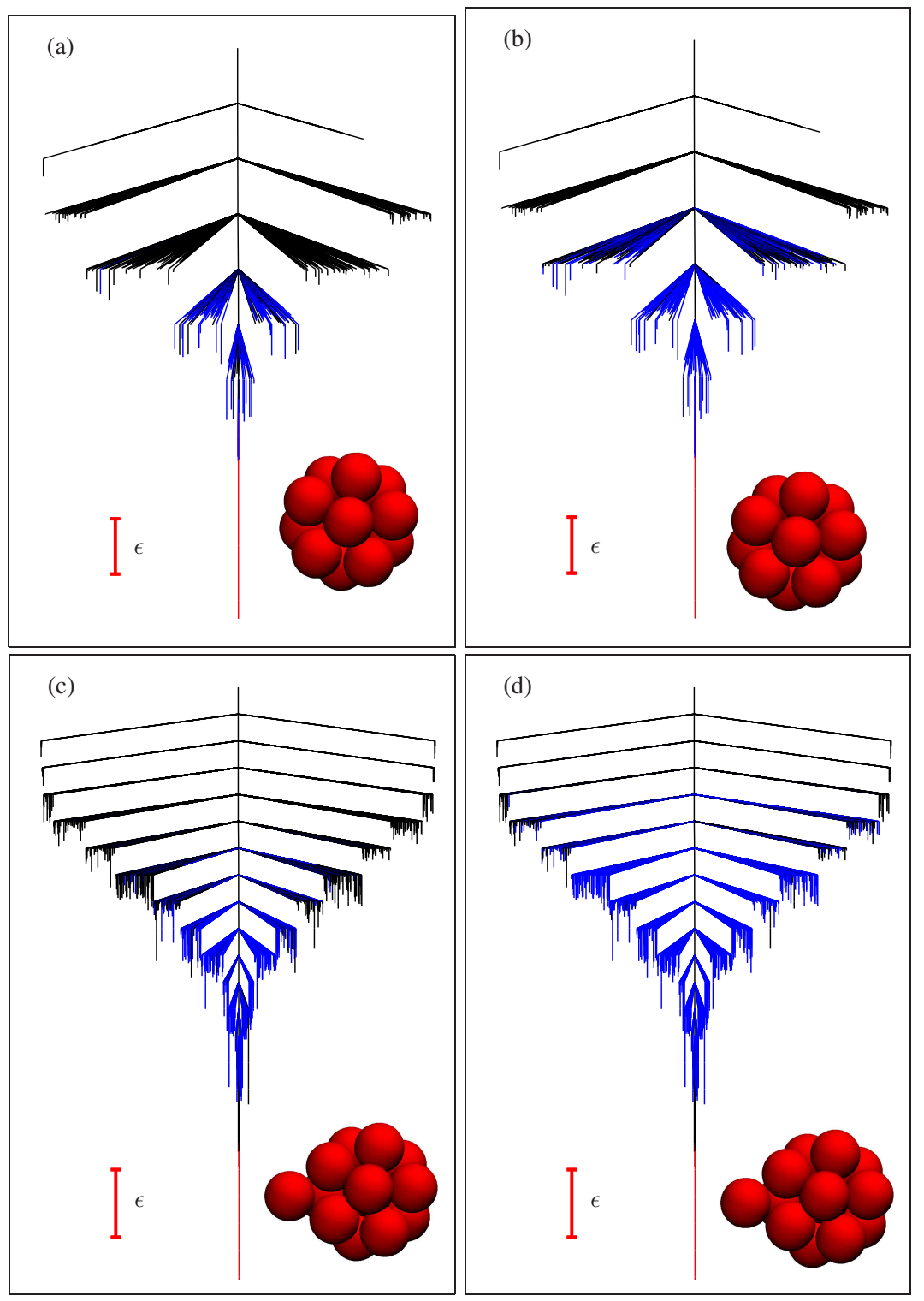

FIG. 2: Disconnectivity graphs for $\mathrm{LJ}_{13}$ coloured according to the occupation probability gradients projected onto local minima, as derived in equation (19). Minima are coloured for contributions to the heat capacity that account for (a) $90 \%$ and (b) $99 \%$ of the contributions from positive (blue) and negative (red) gradients at $k_{B} T / \epsilon=0.28$. The two-dimensional probability distributions required were obtained by basinsampling [24]. For comparison, the results for $\mathrm{LJ}_{14}$ in panels (c) and (d) correspond to assignments based on harmonic superposition analysis for the occupation probabilities of local minima [equation (12)] with the same thresholds of (c) $90 \%$ and (d) $99 \%$. The global minima are illustrated by the corresponding branches in each case. The corresponding stationary point databases include 1441 minima and 21161 transition states for $\mathrm{LJ}_{13}$, with 3413 minima and 52005 transition states for $\mathrm{LJ}_{14}$. 


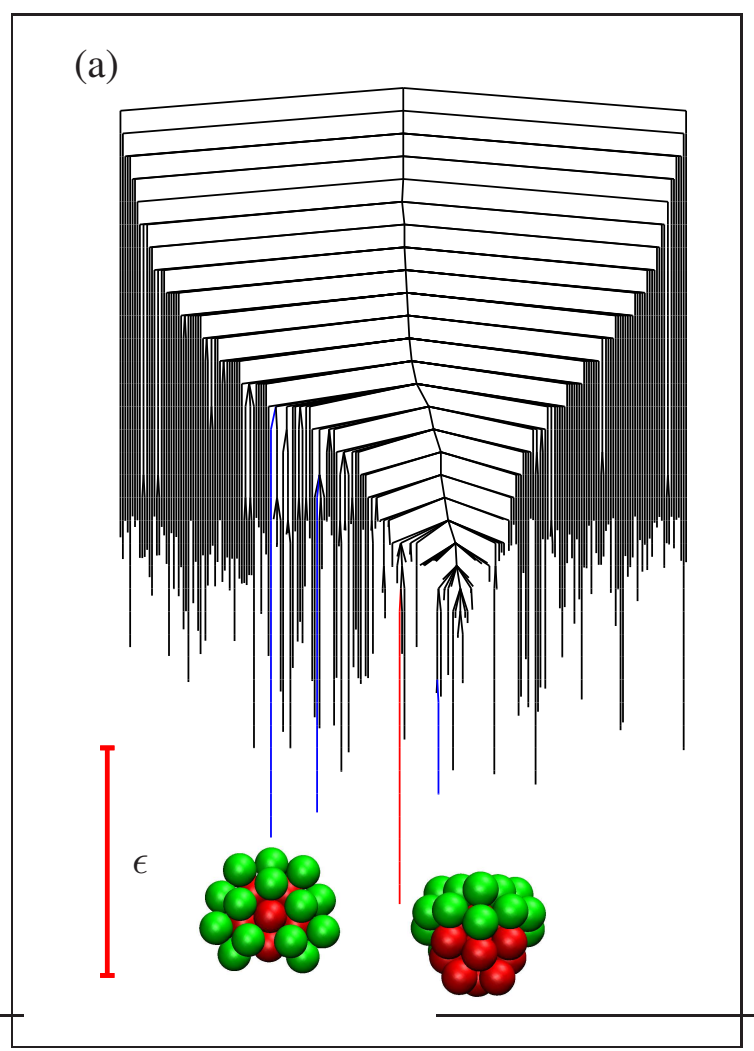

(b)

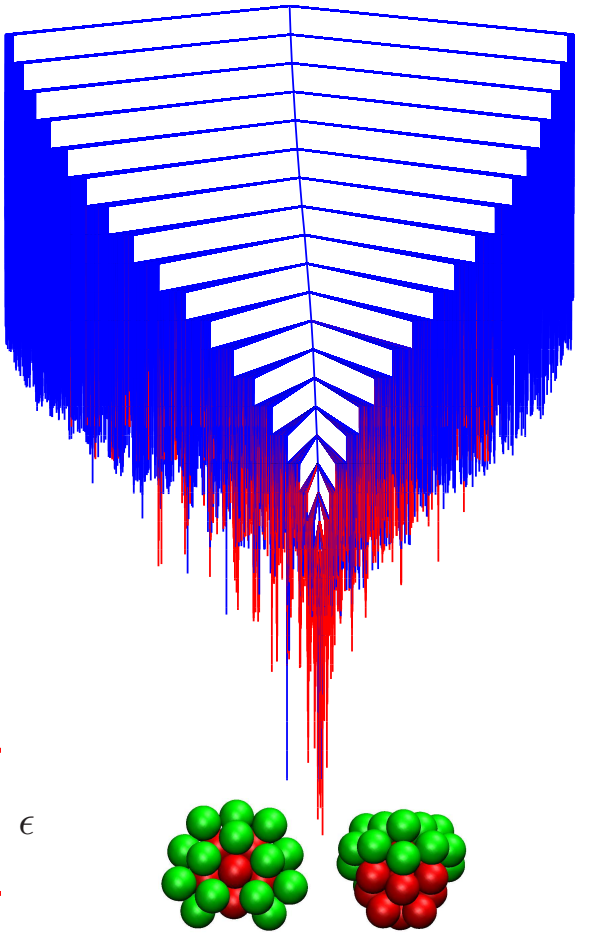

(c)

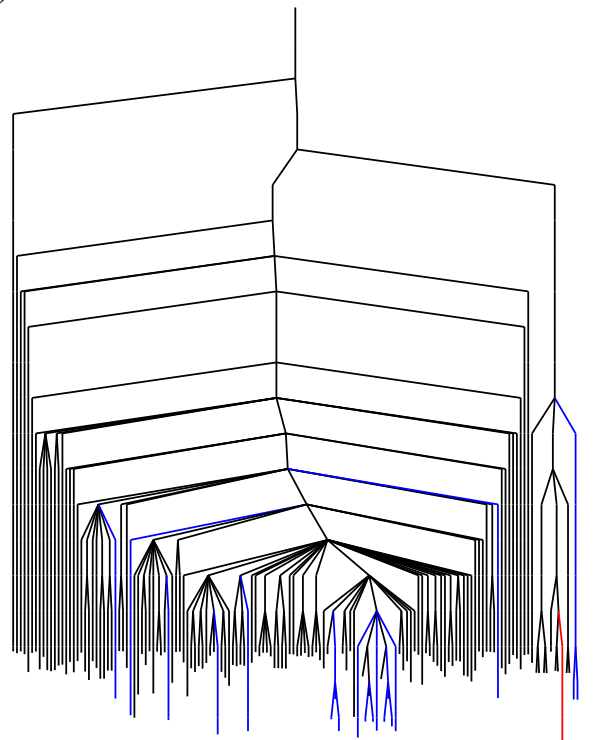

(d)
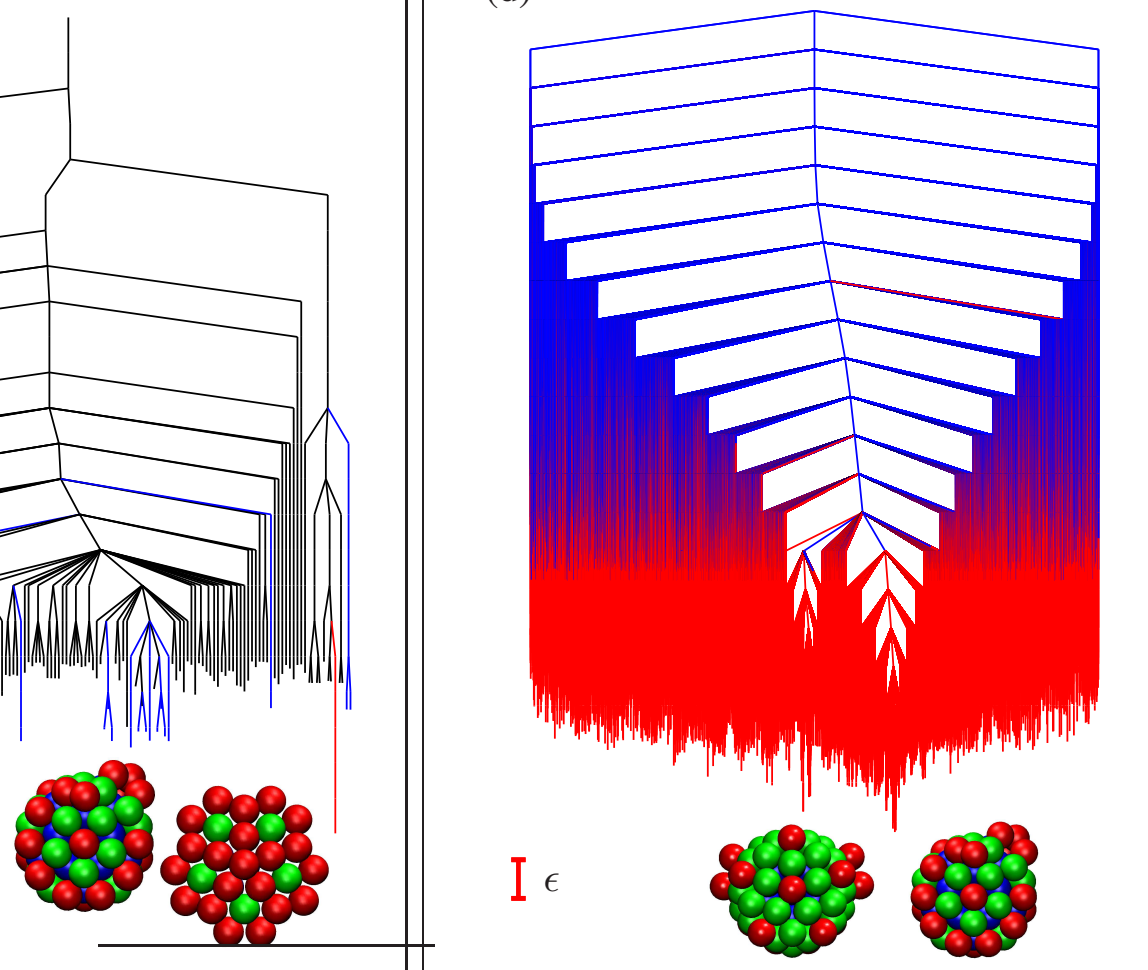

FIG. 3: 
FIG. 3: Disconnectivity graphs for double-funnel landscapes coloured according to the overall occupation probability gradients for the quench energy bin associated with each local minimum, as derived in equation (19). Minima are coloured for contributions to the heat capacity that account for $99 \%$ of the contributions from positive (blue) and negative (red) gradients. The two-dimensional probability distributions required were obtained by basin-sampling [24] and the corresponding stationary point databases include 47193 minima and 100704 transition states for $\mathrm{LJ}_{31}$, with 312178 minima and 602580 transition states for $\mathrm{LJ}_{75}$. (a) $\mathrm{LJ}_{31}$ at $k_{B} T / \epsilon=0.0268$, (b) $\mathrm{LJ}_{31}$ at $k_{B} T / \epsilon=0.329$, (c) $\mathrm{LJ}_{75}$ at $k_{B} T / \epsilon=0.082$, (d) $\mathrm{LJ}_{75}$ at $k_{B} T / \epsilon=0.291$. For the low temperature peaks corresponding to solid-solid transitions the assignments are the same as for the harmonic superposition analysis applied directly to the occupation probabilities of local minima, as defined in equation (12). For the higher temperature melting peaks neglect of well anharmonicity in a harmonic analysis systematically shifts positive probability gradients to higher energy minima (not shown). The lowest and second-lowest minima with Mackay and anti-Mackay overlayers [28] are illustrated for $\mathrm{LJ}_{31}$ in panels (a) and (b). For $\mathrm{LJ}_{75}$ the global minimum Marks decahedron and the second-lowest minimum based on icosahedral packing with an anti-Mackay overlayer are illustrated in panel (c). The graphs in panels (b) and (d) exclude all minima that do not contribute to components of the heat capacity peak within the $99 \%$ threshold, and the global minimum does not appear for $\mathrm{LJ}_{75}$ in (d). Instead the lowest minimum based on icosahedral packing with a Mackay overlayer is illustrated along with the second-lowest minimum, both of which belong to the set with negative occupation probability gradient. 

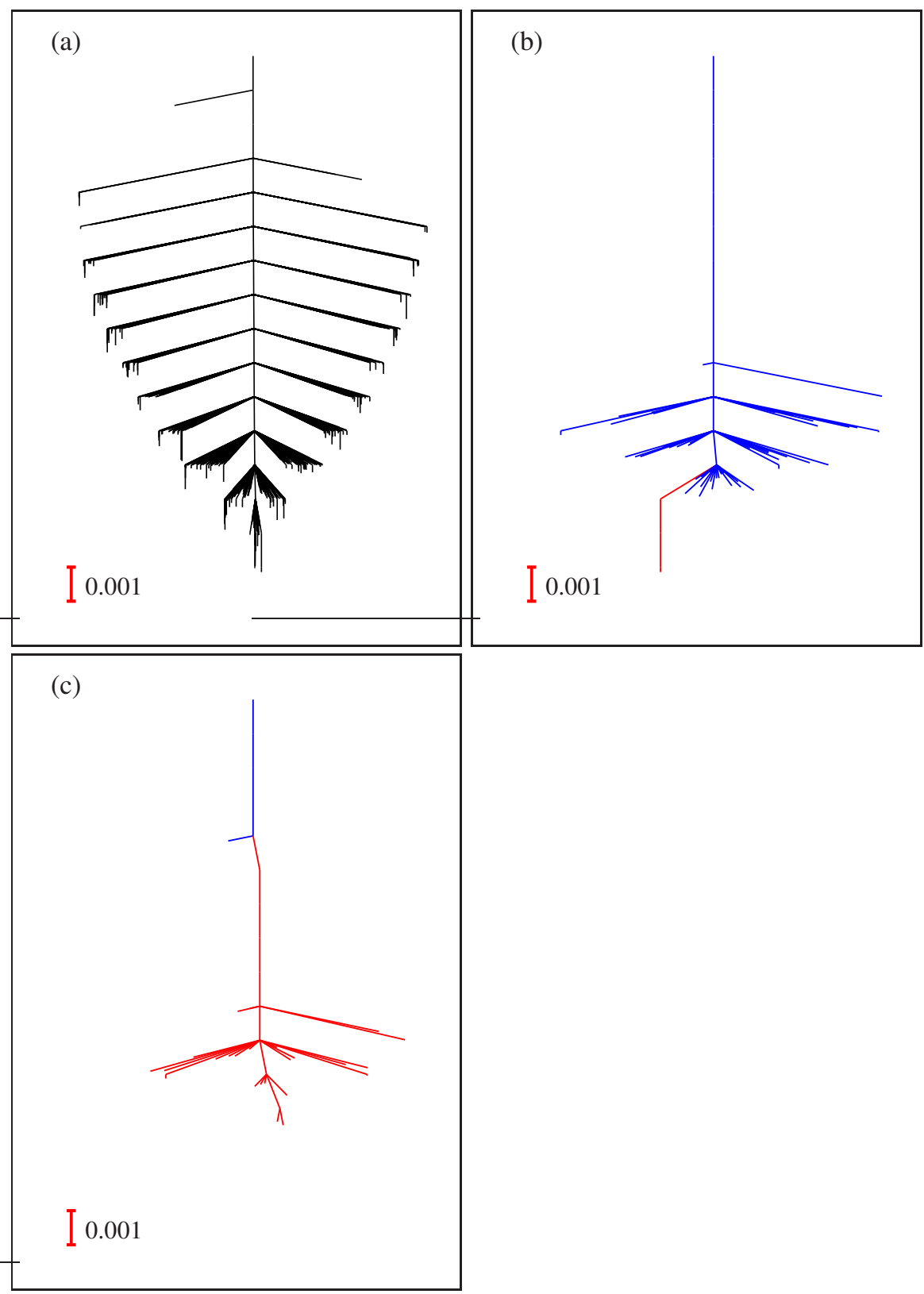

FIG. 4: Disconnectivity graphs for a machine learning landscape corresponding to the heat capacity analogue illustrated in Figure 1b. Panel (a) is the complete graph including all the minima. Panels (b) and (c) correspond to the temperatures of the two peaks in $C_{V}$ at (b) $k_{B} T=0.000236$, (b) $k_{B} T=0.000761$, including only minima that account for $90 \%$ of the contributions from positive (blue) and negative (red) gradients based on harmonic superposition analysis for the occupation probabilities [equation (12)]. The vertical position of each minimum is the same in the three panels and corresponds to the value of the cost function. The stationary point database contains 1997 minima and 7492 transition states. 